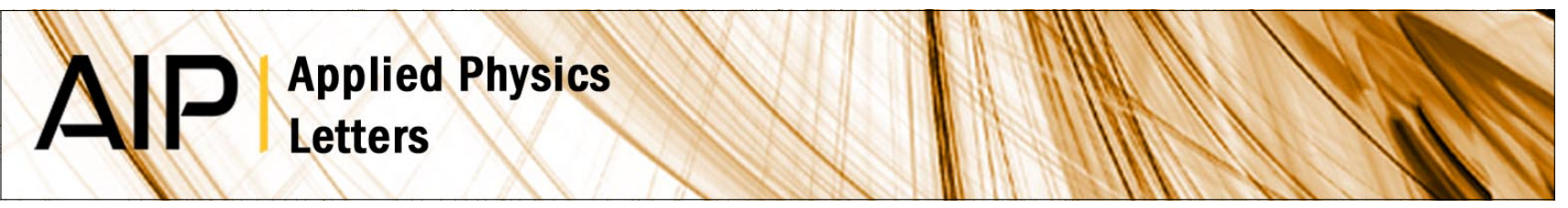

\title{
Effects of elastic and inelastic interactions on phase contrast images in tapping-mode scanning force microscopy
}

Javier Tamayo and Ricardo García

Citation: Appl. Phys. Lett. 71, 2394 (1997); doi: 10.1063/1.120039

View online: http://dx.doi.org/10.1063/1.120039

View Table of Contents: http://apl.aip.org/resource/1/APPLAB/v71/i16

Published by the American Institute of Physics.

\section{Related Articles}

Design and performance of a combined secondary ion mass spectrometry-scanning probe microscopy instrument for high sensitivity and high-resolution elemental three-dimensional analysis

Rev. Sci. Instrum. 83, 063702 (2012)

High-speed Lissajous-scan atomic force microscopy: Scan pattern planning and control design issues Rev. Sci. Instrum. 83, 063701 (2012)

High potential sensitivity in heterodyne amplitude-modulation Kelvin probe force microscopy

Appl. Phys. Lett. 100, 223104 (2012)

Effect of multiplicative noise on least-squares parameter estimation with applications to the atomic force microscope

Rev. Sci. Instrum. 83, 055106 (2012)

Retrofitting an atomic force microscope with photothermal excitation for a clean cantilever response in low $Q$ environments

Rev. Sci. Instrum. 83, 053703 (2012)

\section{Additional information on Appl. Phys. Lett.}

Journal Homepage: http://apl.aip.org/

Journal Information: http://apl.aip.org/about/about_the_journal

Top downloads: http://apl.aip.org/features/most_downloaded

Information for Authors: http://apl.aip.org/authors

\section{ADVERTISEMENT}

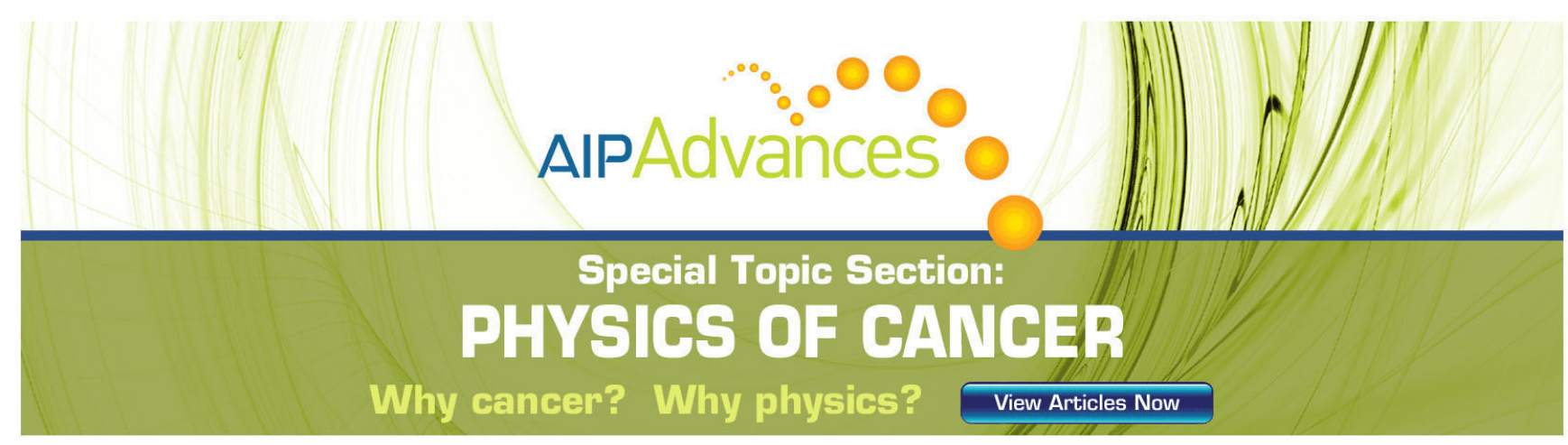




\title{
Effects of elastic and inelastic interactions on phase contrast images in tapping-mode scanning force microscopy
}

\author{
Javier Tamayo and Ricardo García ${ }^{\text {a) }}$ \\ Instituto de Microelectrónica de Madrid, CNM, CSIC, Isaac Newton 8, 28760 Tres Cantos, Madrid, Spain
}

(Received 2 June 1997; accepted for publication 22 July 1997)

\begin{abstract}
The dependence of phase contrast in tapping-mode scanning force microscopy on elastic and inelastic interactions is studied. The cantilever-tip ensemble is simulated as a driven, damped harmonic oscillator. It is found that for tip-sample elastic interactions, phase contrast is independent of the sample's elastic properties. However, phase contrast associated with elastic modulus variations are observed if viscous damping or adhesion energy hysteresis is considered during tip-sample contact. The phase shift versus tip-sample equilibrium separation was measured for a compliant material (polypropylene) and for a stiff sample (mica). The agreement obtained between theory and experiment supports the conclusions derived from the model. These results emphasize the relevance of energy dissipating processes at the nanometer scale to explain phase contrast imaging in tapping-mode force microscopy. (C) 1997 American Institute of Physics.
\end{abstract}

[S0003-6951(97)04838-9]

The scanning force microscope (SFM) has become a versatile and powerful tool for imaging and modification of a wide range of materials such as biomolecules, polymers, metals, and semiconductors. ${ }^{1,2}$ To minimize tip-sample deformation and, in some cases, to improve spatial resolution, several dynamic modes have been developed. ${ }^{3-6}$ In the tapping mode, ${ }^{4}$ the cantilever-tip ensemble is oscillated at a frequency close to its resonance. At one end of each cycle the tip strikes the sample. The substantial reduction of the lateral force exerted on the sample, in comparison with contact SFM, explains its ability to image very compliant materials. $^{7}$

Recently, it was proposed that recording the difference between the phase angle of the excitation signal and the deflection of the cantilever could be applied for performing compositional maps and for imaging material properties. ${ }^{7-9}$ The difference in phase angles is called phase shift, and the images recording phase shifts during tapping operation are called phase contrast images. Phase contrast images of liquid droplets, ${ }^{7}$ polymer patches deposited on silicon, ${ }^{10}$ and polymer blends ${ }^{11}$ were also reported.

Several theoretical models have been developed to explain some of the relevant parameters of dynamic force microscopy such as sample deformation, ${ }^{7,9}$ contact times, ${ }^{7}$ phase angles, ${ }^{7,9}$ amplitudes, ${ }^{12-15}$ and applied forces. ${ }^{12,14}$ However, the specific sample properties that gave rise to phase contrast during tapping operation remained unclear. It was suggested that phase contrast images of heterogeneous samples are related to surface stiffness variations associated with elastic modulus changes. ${ }^{10}$

In this letter, we study the influence of elastic properties on phase contrast in tapping operation. It was found that in the absence of damping, phase contrast was insensitive to variations of the sample elastic properties. However, phase contrast due to variations of elastic properties arose if the sample had internal degrees for energy dissipation. We con-

\footnotetext{
a) Author to whom correspondence should be addressed. Electronic mail: rgarcia at imm.cnm.csic.es
}

sidered two channels for energy dissipation, viscous damping and adhesion energy hysteresis. The good agreement obtained between experiment and theory for mica and polypropylene emphasizes the relevance of energy dissipating processes to obtain phase contrast imaging.

The dynamics of the cantilever-tip ensemble is described by a nonlinear, second-order differential equation,

$$
m \frac{d z^{2}}{d^{2} t}=-k_{c} z-\frac{m w_{0}}{Q} \frac{d z}{d t}+F_{\text {int }}+F_{\nu}+F_{0} \cos w t
$$

where $F_{0}$ and $\omega$ are the amplitude and angular frequency of the driving force, respectively; $Q, \omega_{0}$, and $k_{c}$ are the quality factor, resonance frequency, and spring constant of the cantilever, respectively. $F_{\text {int }}$ denotes the tip-sample interaction. Attractive forces (van der Waals) were simulated by the interaction of a sphere with a flat surface, and repulsive forces were calculated assuming an hertzian contact. ${ }^{7}$ The sample viscous response to the tip movement is calculated by

$$
F_{\nu}=-\eta \sqrt{R \delta} \frac{d z}{d t},
$$

where $\eta$ is the sample viscosity, $R$ is the tip's radius, and $\delta$ is the sample deformation. In this model, the sample is characterized by its elastic and viscosity coefficients. We also considered the effect of adhesion energy hysteresis on phase shifts. This concept is derived from boundary friction studies. ${ }^{16,17}$ The hysteresis was calculated by assuming different adhesion forces between approaching and retracting cycles. ${ }^{18}$ Phase shifts are obtained by solving numerically Eq. (1). Experiments and calculations are performed at the cantilever's free resonant frequency.

In Fig. 1, the phase shift is calculated as a function of the elastic properties of the sample for three situations. (i) Elastic interactions, (ii) an interaction that includes viscous damping, and (iii) an interaction that involves adhesion energy hysteresis. In the absence of energy dissipating processes (open circles), the phase shift is independent of the sample elastic modulus over the four order of magnitude range. Compliant biomolecules and polymers $(\sim 0.5 \mathrm{GPa})$ and stiff 


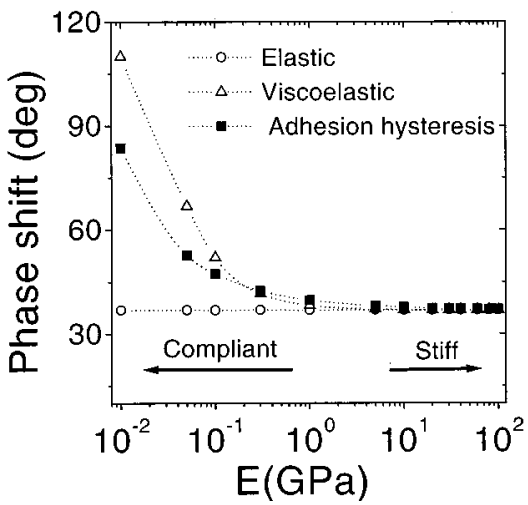

FIG. 1. Theoretical phase shift dependence on elastic properties for several tip-sample interactions. Elastic interactions (open circles); for a tip-sample interaction with viscous damping $\eta=30 \mathrm{~Pa}$ s (triangles); for a tip-sample interaction with adhesion energy hysteresis, $\gamma_{A}=10 \mathrm{~mJ} \mathrm{~m}^{-2}, \quad \gamma_{R}$ $=60 \mathrm{~mJ} \mathrm{~m}^{-2}$ (squares). $A_{t} / A_{0}=0.6, Q=500, \quad k_{c}=20 \mathrm{~N} / \mathrm{m}$, and $V_{t}$ $=200 \mathrm{kHz}$.

materials such as silicon $(\sim 130 \mathrm{GPa})$ fall within that range. The initial phase shift of $36.87^{\circ}$ is set by the tapping conditions.

Stiff samples show, for the same tip-sample equilibrium separation, higher repulsive forces than compliant samples or regions. Consequently, stiff and compliant regions should behave differently. This seems at variance with the above results. The independence of phase contrast with elastic properties changes is a property of the tapping operation. There, feedback parameter is the tapping amplitude $\left(A_{t}\right) . A_{t}$ is obtained by adding the tip-sample equilibrium separation $z_{c}$ and the sample deformation $\delta$.

Now, let's assume a sample with two contiguous regions of Young's modulus $E_{1}$ and $E_{2}$, respectively. In the tapping operation, $A_{t}$ is kept constant, then

$$
A_{t}=z_{c 1}+\delta_{1}=z_{c 2}+\delta_{2} .
$$

The sample deformation is smaller in the stiffer region, as a consequence $z_{c 1}>z_{c 2}$. This change in separation between regions $E_{1}$ and $E_{2}$ compensates the contribution associated with $E$ differences. The net effect is that in the tappingmode, phase shifts are independent of variations of the elastic modulus.

The presence of viscous damping or adhesion energy hysteresis, however, alters the above behavior considerably. The phase shift decreases with the sample's stiffness. This effect is enhanced in compliant samples (below $1 \mathrm{Gpa}$ ), in which changes of $E$ by a factor 2 may produce phase-shifts variations of several degrees, i.e., well above the experimental resolution of $0.1^{\circ}$. The value of the phase shift depends on the details of the energy dissipating process, but the shapes of the curves are quite similar for the cases simulated here.

The effect of changing the strength of the adhesion force was also investigated. In the absence of adhesion energy hysteresis, the phase shift was insensitive to adhesion force variations.

To illustrate some of the basic features of phase shifts in dynamics SFM and to test the model, we performed experiments on two materials with different mechanical properties, mica and polypropylene (PP).

Figure 2 shows the experimental dependence of the

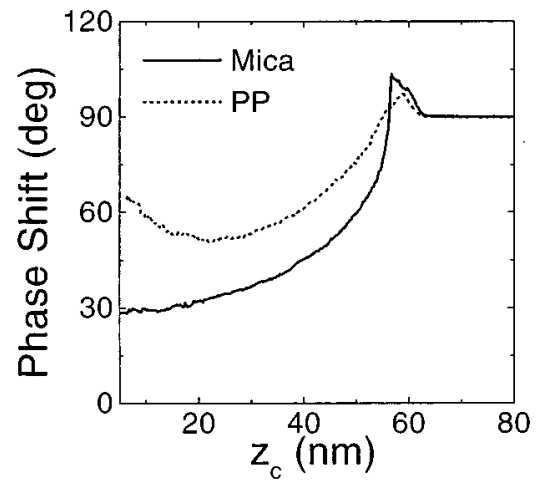

FIG. 2. Experimental phase-shift dependence on tip-sample equilibrium separation curves for mica and polypropylene. Free amplitude $A_{0}=62 \mathrm{~nm}$, $\nu_{t}=345.83 \mathrm{kHz}, Q=392$, and $k_{c}=40 \mathrm{~N} / \mathrm{m}$.

phase shift on the tip-sample equilibrium separation for mica and PP (Celgard 2400, Hoecht-Celanese). For separations larger than the free amplitude, no appreciable phase shifts are observed.

When the tip is very close to the sample surface, an increase of the phase of about $13^{\circ}$ is observed for mica. The increase is of $7^{\circ}$ for the PP. There, the interaction potential is dominated by attractive forces. The positive gradient of the force shifts the cantilever resonance frequency to lower values. This, in turn, shifts the phase upwards. Once there is tip-sample contact, the phase shift decreases with decreasing separation due to the negative gradient of the interaction force.

Three major differences between the mica and the PP curves are observed. (1) The transition between noncontact and intermittent contact produces a sudden decrease of the phase for mica, while in the polymer there is a smooth variation. (2) For mica, the phase shift between the starting and final position is $61^{\circ}$. In the polymer, the maximum phaseshift variation is about $39^{\circ}$. (3) The polymer also shows an increase of the phase for tip-sample equilibrium separations smaller than $20 \mathrm{~nm}$.

Figure 3(a) shows the calculations for mica with and without adhesion energy hysteresis. We assumed that $\eta$ $=0.0 \mathrm{~Pa} \mathrm{~s}$ and $E=24.5 \mathrm{GPa}$ (Ref. 19). The calculations reproduce the sharp transition observed experimentally between noncontact and intermittent contact. The effect of adhesion energy hysteresis is more noticeable for small tipsample separations, where adhesion forces may become dominant.

In Fig. 3(b) are plotted the calculations for the polymer. The mechanical properties of the polypropylene were taken from dynamic measurements at frequencies similar to those used here, $E=0.5 \mathrm{GPa}$ and $\eta=30 \mathrm{~Pa}$ s (Ref. 20). The combined effect of viscous damping and adhesion energy hysteresis reproduces the gradual phase-shift transition from noncontact to intermittent contact observed experimentally as well as the existence of a minimum. There is also a reasonable quantitative agreement between experiment and theory. The differences obtained between mica and PP in the noncontact region are associated with the strength of the attractive force. This is smaller in the polymer.

The above results emphasize the role of energy dissipation in the sample to obtain phase contrast images during the 

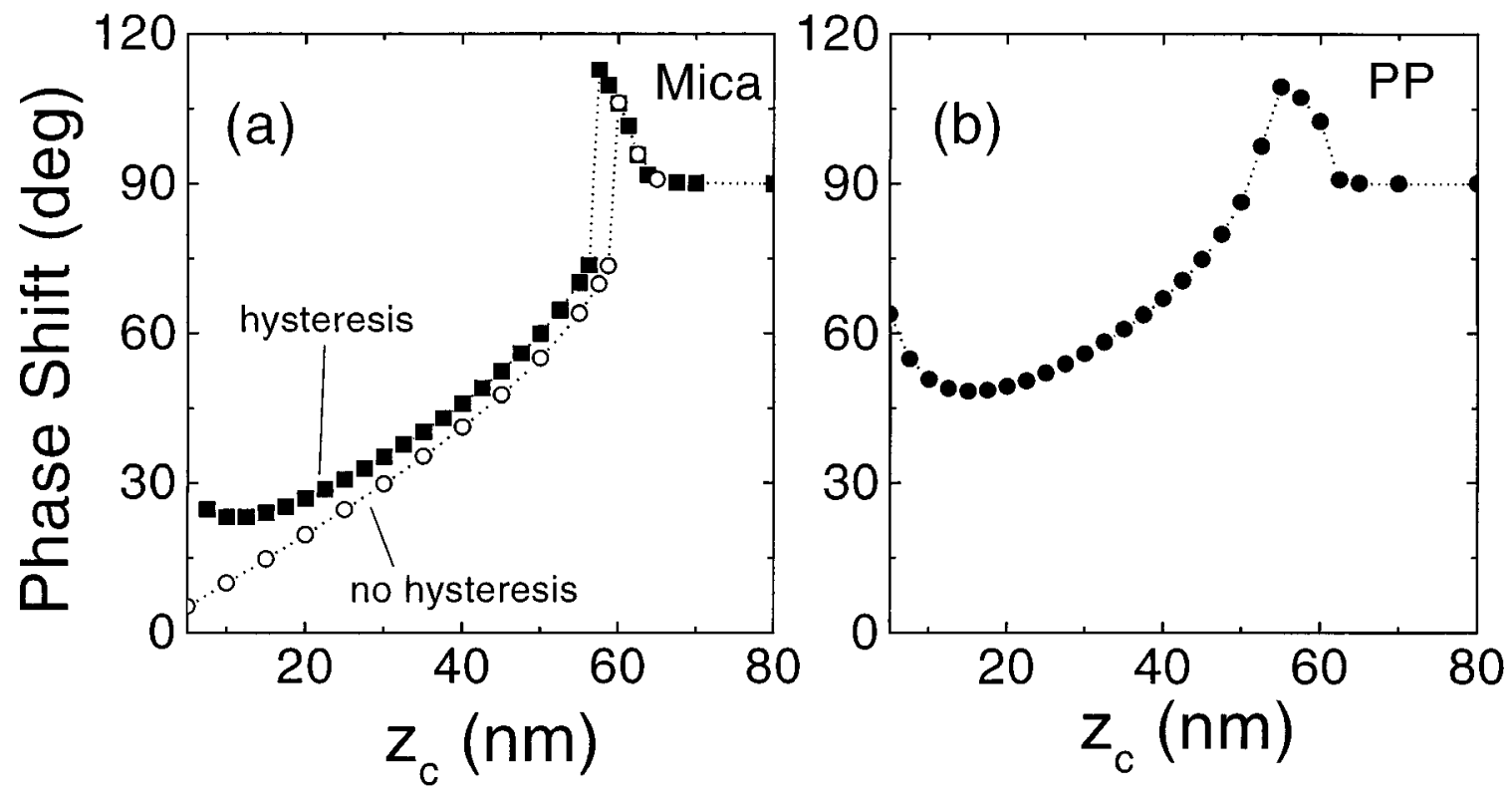

FIG. 3. (a) Simulation for mica without adhesion energy hysteresis (open circles) and with hysteresis $\gamma_{A}=150 \mathrm{~mJ} \mathrm{~m}^{-2}, \gamma_{R}=225 \mathrm{~mJ} \mathrm{~m}^{-2}$ (squares). (b) Simulation for polypropylene, $\eta=30 \mathrm{~Pa} \mathrm{~s}, \gamma_{A}=10 \mathrm{~mJ} \mathrm{~m}^{-2}$, and $\gamma_{R}=60 \mathrm{~mJ} \mathrm{~m}^{-2}$. Both cases with $\nu_{t}=345.83 \mathrm{kHz}, Q=392, A_{0}=62 \mathrm{~nm}$, and $k_{c}=40 \mathrm{~N} / \mathrm{m}$.

tapping operation. Phase contrast imaging may arise from differences in elastic properties once the sample has a channel for interacting inelastically with the tip.

These results underline the complex behavior of phase shifts in dynamic force microscopy where elastic and inelastic processes alike may be involved. They also open new ways to study relevant interfacial phenomena such as friction, and more generally, energy dissipation at the nanometer scale.

This work has been supported by Dirección General de Investigación Científica y Técnica of Spain (DGICYT) (PB94-0016).

${ }^{1}$ D. Rugar and P. Hansma, Phys. Today 43, 23 (1990).

${ }^{2}$ C. Bustamante and D. Keller, Phys. Today 48, 32 (1995).

${ }^{3}$ Y. Martin, C. C. Williams, and H. K. Wickramasinghe, J. Appl. Phys. 61, 4723 (1987).

${ }^{4}$ Q. Zhong, D. Imniss, K. Kjoller, and V. B. Elings, Surf. Sci. 290, L688 (1993).

${ }^{5}$ D. Anselmetti, R. Lüthi, E. Meyer, T. Richmond, M. Dreier, J. E. Frommer, and H.-J. Güntherodt, Nanotechnology 5, 87 (1994).

${ }^{6}$ F. J. Giessibl, Science 267, 68 (1995).

${ }^{7}$ J. Tamayo and R. García, Langmuir 12, 4430 (1996).
${ }^{8}$ D. A. Chernoff, Proceedings Microscopy and Microanalysis 1995 (Jones and Begell, New York, 1995).

${ }^{9}$ R. G. Winkler, J. P. Spatz, S. Sheiko, M. Möller, R. Reineker, and O. Marti, Phys. Rev. B 54, 8908 (1996).

${ }^{10}$ S. N. Magonov, V. Elings, and M.-H. Whangbo, Surf. Sci. 375, L385 (1997).

${ }^{11}$ G. Bar, Y. Thomann, R. Brandsch, H.-J. Cantow, and M.-H. Whangbo, Langmuir (in press)

${ }^{12}$ J. Chen, R. K. Workman, D. Sarid, and R. Höper, Nanotechnology 5, 199 (1994); D. Sarid, T. G. Russel, R. K. Workman, and D. Chen, J. Vac. Sci. Technol. B 14, 864 (1996).

${ }^{13}$ G. Y. Chen, R. J. Warmack, A. Huang, and T. Thundat, J. Appl. Phys. 78, 1465 (1995)

${ }^{14}$ B. Anczykowski, D. Krüger, and H. Fuchs, Phys. Rev. B 53, 15485 (1996)

${ }^{15}$ N. A. Burnham, O. P. Behrend, F. Oulevey, G. Gremaud, P.-J. Gallo, D. Gourdon, E. Dupas, A. J. Kulik, H. M. Pollock, and G. A. D. Briggs, Nanotechnology 8, 67 (1997).

${ }^{16}$ H. Yoshizawa, Y.-L. Chen, and J. Israelachvili, J. Phys. Chem. 97, 4128 (1993)

${ }^{17}$ M. K. Chaudhury, Mater. Sci. Eng. R. Rep. 16, 97159 (1996).

${ }^{18}$ R. García, J. Tamayo, M. Calleja, and F. García (unpublished).

${ }^{19}$ L. E. McNeil and M. Grimsditch, J. Phys.: Condens. Matter 5, 1681 (1992).

${ }^{20}$ F.-J. Wartmann and K. V. Schultz, Polymer 36, 315 (1995). 